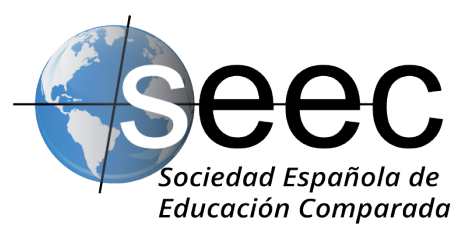

\title{
Evaluación de la práctica de los docentes de educación obligatoria, estudio comparado en Europa
}

Teacher evaluation in European Compulsory education

\section{Susana Herradas Martín*}

DOI: $10.5944 /$ reec.39.2021.27388

Recibido: 29 de abril de 2020
Aceptado: 7 de julio de 2020

*Susana Herradas Martín: directora del CPT Virgen de los Remedios de Colmenar Viejo, experiencia como Jefa de Estudios y Secretaria en equipos directivos de centros de Infantil y Primaria; maestra de Primaria especialista en Educación Física por la Universidad Complutense de la Comunidad de Madrid; Máster de Calidad y Mejora en Educación por la Universidad Autónoma de Madrid. Datos de contacto: E-mail: susana.herradas@educa.madrid.org 


\title{
Resumen
}

El análisis de la evaluación docente constituye una pieza clave en la mejora del proceso de enseñanza-aprendizaje y del desarrollo profesional docente. El Marco Europeo ha definido una línea de trabajo sobre las competencias docentes que intentan dar respuesta a las necesidades de la sociedad actual. ¿Cuáles son estas competencias y cómo se evalúan dentro de la práctica docente? Los diferentes países europeos diseñan sus políticas de evaluación educativa, seleccionados cuatro ejemplos representativos de modelos educativos (centroeuropeo, nórdico, anglosajón y meridional) se realizará un estudio comparado sobre evaluación docente. En una primera fase se elaborará la unidad de análisis incluyendo las siguientes dimensiones: el carácter de la evaluación, la evaluación de la práctica docente, desarrollo profesional docente, recogida de datos de la evaluación docente y por último la devolución de los resultados de la evaluación y su uso. Se aplicará la unidad de análisis a cada uno de los sistemas educativos del estudio con ayuda de un árbol de parámetros. Se observa que no hay un modelo unificado de evaluación docente en los cuatro países del estudio. En los diferentes modelos de evaluación analizados no se recogen de forma detallada las competencias docentes propuestas por la Comisión Europea para la mejora de la práctica docente. Sí se observa un creciente interés por parte de los diferentes sistemas educativos de implementar la evaluación docente como elemento para conseguir la eficacia educativa. De los modelos estudiados los que presentan mejores resultados son aquellos que son capaces de generar una cultura evaluativa que facilite el desarrollo de un sistema dinámico y flexible donde los resultados reviertan directamente en los alumnos, los docentes y la institución a la que pertenecen.

Palabras clave: evaluación docente; educación obligatoria; competencias docentes.

\begin{abstract}
The analysis of teacher evaluation constitutes a key piece in improving the teaching-learning process and teacher professional development. The European Framework has defined a line of work on teaching competencies that try to respond to the needs of today's society. What are these competences and how are they evaluated within teaching practice? Different European countries design their educational evaluation policies from different perspectives. Selected four representative examples of educational models (Central European, Nordic, Anglo-Saxon, and Southern) of them, a comparative study on teacher evaluation will be carried out, through which this article will try to answer the questions asked. In the first phase, the unit of analysis will be elaborated including the following dimensions: the nature of the evaluation, the assessment of teaching practice, teacher professional development, collection of data from the teaching evaluation, and finally the return of the evaluation results. The unit of analysis will be applied to each of the educational systems with the help the tree of parameters. There is no unified model of teacher evaluation in the four countries. In the different evaluation models analyzed, the teaching competences proposed by the European Commission are not detailed. There is a growing interest on the part of the different educational systems to implement teacher assessment as an element to achieve educational effectiveness. Of the models studied, the best results are the systems with an evaluative culture.
\end{abstract}

Keywords: teacher evaluation; compulsory education; teacher competencies. 


\section{Introducción}

Desde la Comisión Europea, en el informe de Eurydice de 2015 que hace referencia a «La Profesión Docente en Europa. Prácticas, percepciones y políticas», se analiza y profundiza en el papel del docente dentro del proceso de aprendizaje del estudiante. La Unión Europea a través de las metas «Educación y Formación 2020» persigue, a pesar de la diversidad de sistemas educativos, que se garantice la transmisión de valores comunes de tolerancia y libertad de expresión dentro de una ciudadanía europea activa que facilite la cohesión social y la integración. Sin olvidar el desarrollo integral del individuo, capacitándolo para desenvolverse en el actual mercado laboral y lograr un desarrollo equilibrado de su personalidad.

Todas son demandas de una sociedad que exige nuevos modelos de aprendizaje, nuevos desarrollos tecnológicos y nuevas prácticas que modifican la práctica docente, influyendo directamente sobre el desarrollo del alumno (OCDE: TALIS, 2018 y PISA; 2012).

La Comisión está trabajando con los Estados miembros en la creación de un Espacio Europeo de Educación de aquí a 2025. Las medidas presentadas en 2018 recogen las competencias clave para alcanzar la calidad de la educación en primaria y secundaria, que ha de ser gratuita, equitativa y de calidad, producir resultados de aprendizajes pertinentes y efectivos, tal como recoge el cuarto de los Objetivos de Desarrollo Sostenible 2030, de la ONU (European Comission, 2019).

La evaluación docente se perfila como un recurso para determinar la mejor práctica, y certificar si dichas competencias están presentes o no en las aulas. El informe Eurydice 2019 sobre la profesión docente en Europa recoge que la evaluación del profesorado busca valorar el rendimiento individual de los docentes y garantizar que estos disponen de las destrezas suficientes para desarrollar su actividad con eficacia (Eurydice, 2019). La evaluación docente es un tema muy controvertido. En 2009 la OCDE publica un informe sobre Evaluación Docente, considerándola como un elemento de mejora del desempeño, del desarrollo profesional y de un incremento de la calidad de la enseñanza (DarlingHammond, 2000), ya que revierte directamente sobre los resultados del aprendizaje de los alumnos. Una evaluación formativa facilita la autoeficacia docente (Bolívar, 2008), componente clave en la búsqueda de políticas docentes efectivas. Una manera de valorar la calidad de la enseñanza es evaluar al profesorado (Lagos, 2004). Esta evaluación docente normalmente se da como un proceso separado de otros procesos de garantía de la calidad de la institución en la que se trabaje (Eurydice, 2019).

En la búsqueda de estas políticas efectivas de evaluación se compararán cuatro modelos europeos. El primer modelo será el de Alemania, un modelo denominado de «regionalización» (Gil, 2006). Son las diferentes regiones (Länder) las que realizan una interpretación administrativa de una norma general. Este sentido de regionalización se parece a la estructura autonómica española. El segundo modelo, es el modelo de Finlandia, que posee una estructura denominada «localización» (Gil, 2006) caracterizada por la gran autonomía de los centros y el control local. El tercer modelo a contrastar sería el modelo de Reino Unido con «una autonomía institucional en cuasi mercado» (Gil, 2006). La financiación de las escuelas depende de la matriculación en ellas y de los resultados del alumnado. Se fomenta una competitividad y un mercado donde los padres tengan libertad para elegir y se dé por hecho la efectividad de la institución. Y el cuarto modelo, el modelo español, que posee una estructura descentralizada, son las Comunidades Autónomas las que asumen las competencias educativas y legislan en base 
a una ley autonómica, eso sí, respetando la ley general común a todo el país.

El presente artículo pretende mostrar el estado de la evaluación docente en diferentes sistemas educativos representativos del panorama europeo.

\subsection{Marco europeo de competencias docente}

Ya en el año 2004 la Comisión Europea publica un informe en el que establece «Los Principios europeos comunes para las competencias docentes y cualificaciones». Estos principios intentan dar respuesta a las necesidades de un nuevo orden social modificado por la era digital que favorece la globalización y los movimientos migratorios. Un cambiante mercado laboral genera un nuevo concepto de formación a lo largo de la vida Lifelong learning (Day, 2002). Dos circunstancias, que provocan una mayor diversidad en las aulas y nuevos retos. Lograr una verdadera integración y una formación competencial que facilite la incorporación a la sociedad, se convierten en desafíos docentes (Carbonell, 2007; Cardús, 2007; Esteve, 2003; Marcelo 2002).

Desde este informe se resalta la necesidad de reflexión del docente sobre los procesos de enseñanza y aprendizaje basados en el conocimiento de la materia, el currículo, las estrategias metodológicas, la innovación, la investigación y las dimensiones sociales y culturales de la educación (Comisión Europea, 2004). Se establecen unos principios comunes que pretenden guiar las diferentes políticas educativas de los países de la Unión para incorporar estos elementos tanto a la formación docente como a su desempeño.

En relación a su formación previa se aboga por una profesión de posgrado, que asegure: conocimiento pedagógico del/as área/s que impartirán, las habilidades y competencias requeridas para guiar y apoyar a los estudiantes, además de un conocimiento de la realidad cultural y social que facilite la integración y el respeto a las diferentes realidades culturales. Esta formación también está enfocada a desarrollar un docente que sea capaz de formarse a lo largo de toda su carrera (DPC) o Lifelong Teacher Education (Valle, y Manso, 2017) que le permita innovar e irse adaptando a las nuevas demandas de una sociedad cambiante. Con este fin se facilita la movilidad del docente, fomentando el intercambio de experiencias y prácticas que le enriquezcan a su vez a los sistemas educativos, así como a los estudiantes.

Los docentes deberían de ser capaces de trabajar simultáneamente estos tres ámbitos del desarrollo de las personas: por un lado con la información, la tecnología y el conocimiento. Por otro, a nivel personal con los estudiantes, los otros docentes. Y por último, con y en la sociedad como entidad formativa a nivel local, regional, nacional, europeo y mundial. Para ello serán capaces de seleccionar los recursos en un contexto concreto, desarrollando sus actividades de forma efectiva y eficiente. En función de estos tres ámbitos los diferentes sistemas educativos de la Unión Europea generan marcos competenciales docentes y su regulación administrativa. Además del tipo de instituciones que se encargan de dicha regulación y evaluación dentro de las diferentes políticas educativas (Sánchez-Tarazaga, 2016).

En 2012 se recogen 28 competencias principales denominadas «Core Competences» englobadas en tres grandes ámbitos. El primero, los conocimientos y su comprensión, seguido de las habilidades para aplicarlos y por último, las disposiciones en el ámbito emocional como: actitudes, creencias, compromiso y valores (Comisión Europea, 2012b). 


\subsection{Evaluación del profesorado dentro de la Unión Europea}

El Enfoque de la Comisión Europea incluye la evaluación como una herramienta que informa sobre el desempeño docente y garantiza que estos posean las destrezas necesarias para llevar a cabo su labor con eficacia (Eurydice, 2018). Esta evaluación promueve la formación profesional continua (DPC). Generando una dinámica de innovación y adaptación del docente a la realidad educativa (Eurydice, 2013 y 2015).

La evaluación según la Comisión Europea constituye un proceso que conduce a mejores métodos de enseñanza, lo que posibilita la mejora continua (OCDE, 2009). El último informe Eurydice 2018 recoge que, aunque la evaluación docente se encuentra regulada en la inmensa mayoría de los sistemas educativos, las administraciones de rango superior rara vez supervisan los resultados y si la administración es local o el propio centro, esto nunca ocurre.

El informe Eurydice de 2018 detalla cinco ámbitos de trabajo: el primero si existe un modelo centralmente regulado, el segundo atiende a quién realiza la evaluación docente. Si es esporádica o regular es el tercer ámbito. Atender a los instrumentos de evaluación para llevarla a cabo y los resultados de esta conforman el cuarto y el quinto. Los grandes interrogantes del presente trabajo son: ¿Cómo constatar que las competencias clave están presentes en los diferentes modelos de evaluación docente que se comparan? ¿Qué prácticas de evaluación docente son más efectivas para el desempeño docente? ¿Qué diferencias se encuentran entre los modelos europeos de evaluación docente?

\section{Metodología}

Los países de la Unión Europea vienen incorporando las directrices de la Comisión Europea sobre evaluación docente en sus sistemas educativos, aunque bien es cierto que cada uno de ellos lo hace de forma única. Probablemente unos con más éxito que otros. Para conocer las similitudes y diferencias se aplicará el método comparado.

El sentido de seguir este enfoque es ampliar la mera comparación, a una búsqueda de mejora común (Caballero et al., 2016), que ayude en la aplicación de procesos evaluativos como elemento clave de la calidad docente. Como fuentes de este estudio se analizarán los documentos elaborados por la Red Eurydice, Comisión Europea y los estudios internacionales TALIS y PISA de la OCDE referentes a evaluación docente y a los sistemas educativos de los cuatro países del estudio. Además se tendrán en cuenta los documentos derivados de dichos informes que se consideren relevantes para el tema.

La primera fase de la investigación con metodología comparada abordó la definición del problema, los presupuestos de partida y la unidad de análisis.

\section{1. objetivos del estudio}

La evaluación debe mostrar al docente como un profesional que evoluciona a lo largo de la vida. Desde el mismo momento que inicia su formación, irá diseñando su carrera profesional de forma dinámica (Calderhead, Denicolo, y Day, 2012). Estará guiado por la búsqueda de la mejora en su práctica profesional, pero no de forma aislada sino enmarcada en la realidad que le rodea y además conectada con otros docentes (DarlingHammond, Wei, Andree, Richardson y Orphanos, 2009), incluyendo las competencias establecidas por la Comisión Europea en 2015. 
Esta concepción es la base para desarrollar una unidad de análisis de la evaluación docente. Como presupuestos de partida se establecen los siguientes: cómo se estructura la evaluación docente bajo el marco de las competencias docentes a nivel europeo en los cuatro sistemas educativos y determinar qué sistemas de evaluación docente son más efectivos.

\section{2. justificación de modelos de sistemas educativos: unidad de análisis}

La unidad de análisis es la evaluación docente dentro de los diferentes sistemas educativos. Alemania, España, Finlandia, y Reino Unido representan al modelo centroeuropeo, modelo latino, modelo nórdico y modelo anglosajón a nivel educativo. Cada uno de ellos aporta elementos estructurales, organizativos y pedagógicos enriquecedores desde el punto de vista de la evaluación docente.

Se ha incluido Reino Unido por su significación en la percepción competencial que tiene de sus docentes, aunque hay que tener en cuenta que desde el 2017 ya no es miembro de la Unión Europea.

Dentro de esta unidad de análisis se han seleccionado las siguientes dimensiones: el carácter de la evaluación, la evaluación de la práctica docente, desarrollo profesional docente, recogida de datos y por último la devolución de los resultados de la evaluación y su uso.

Una propuesta de marco conceptual la facilita Isoré (2009) en el estudio de la evaluación en los diferentes países de la OCDE. Al configurar el árbol de parámetros se han tenido en cuenta los siguientes aspectos: una evaluación sumativa teniendo en cuenta que las prácticas que se desarrollan mejoren los aprendizajes de los alumnos. Otra formativa referida al desarrollo profesional docente y sus consecuencias en la vida laboral y los actores involucrados en llevarla a cabo.

Las responsabilidades de los docentes se agrupan según Isoré (2009) en cuatro ámbitos del desempeño docente: planificación y preparación referidas al dominio del contenido y los aspectos pedagógicos. El entorno del aula, qué recursos para favorecer un clima de respeto y simpatía que fomente una dinámica de aprendizaje. El desarrollo de relaciones equilibradas y empáticas que faciliten la implicación del alumno en su propio aprendizaje. Aplicar las responsabilidades profesionales: tener presente en la práctica del ejercicio de la reflexión sobre la enseñanza y su mejora. Así como la relación con las familias y demás comunidad educativa para su crecimiento y mejora.

Tomando como referencia el informe TALIS 2018, el éxito de los sistemas de evaluación del profesorado depende de la preparación del proceso de evaluación y las fases de seguimiento del mismo. Así como de la formación y experiencia que deben de tener los evaluadores y de cómo incorporar los resultados de la evaluación al sistema. La elaboración del árbol de parámetros ha tenido en cuenta esta fuente de información (TALIS, 2018).

\section{3. árbol de parámetros de la evaluación docentes: dimensiones- parámetros-indicadores}

El primer aspecto a tener en cuenta para acercarse a la unidad de análisis es el carácter de la evaluación docente (Eurydice, 2018). Dentro de esta dimensión el primer parámetro analizado es la obligatoriedad o voluntariedad. El segundo, al grupo de docentes que participan en la evaluación, se podría pensar que la evaluación docente es generalizada, pero no es así. En ocasiones depende del tipo de contrato, o de una determinada etapa de la carrera profesional; otras atiende al caso de programas generales o programas vocacionales (educación de adultos). 
Podemos dividirla en interna o externa, en función de la naturaleza. La interna se caracteriza porque es la propia escuela la que elabora sus instrumentos de evaluación y los resultados de esta evaluación revierten en ella. En contraposición la externa aplica unos instrumentos estandarizados por las diferentes administraciones educativas.

El último parámetro es la frecuencia con la que esta tiene lugar. Se puede diferenciar una realizada a intervalos regulares, otra realizada en etapas clave de la carrera del docente (promoción) y por último en ocasiones específicas, por ejemplo: al final de un periodo de pruebas o para la contratación.

La segunda dimensión analizada ha sido la práctica docente. Se han tenido en cuenta las Core Competences del marco de regulación europeo (Comisión Europea, 2012b).

El primer parámetro abordado es la planificación y preparación, los indicadores que recogen la información es si la evaluación docente refleja los objetivos de la enseñanza, el conocimiento del currículo por el docente y las competencias pedagógicas (didáctica) del área que imparte.

Le sigue la evaluación del proceso enseñanza-aprendizaje. Si se incluyen diferentes modelos de evaluación: evaluación entre pares, coevaluación, autoevaluación y con diferentes instrumentos de recogida de información del proceso de aprendizaje del alumno, con la participación o no de la administración docente, etc.

El tercero se refiere al clima en el aula. Los indicadores que recoge el árbol de parámetros son los siguientes: tener en cuenta un marco multicultural y multilingüe al crear el ambiente del aula (TALIS, 2018), una cultura de aprendizaje desde un enfoque competencial, cómo se gestiona el ambiente del aula y la estructuración del espacio físico.

Atender a la metodología que aplica el docente, si favorece el aprendizaje individualizado involucrando al alumno. Si se plantea el aprendizaje cooperativo. Si se desarrollan las competencias transversales aumentando la complejidad, para fomentar el desarrollo de la creatividad. Y todo ello incorporando las competencias digitales como herramienta en el desarrollo del propio aprendizaje. Sin olvidarnos de la participación en proyectos de innovación y la investigación en el aula (TALIS, 2018).

La práctica docente debe estar asentada en un principio de equidad, siendo un elemento compensador del contexto o características del alumno. Por esta razón el cuarto parámetro de análisis se basa en cómo el docente incorpora las necesidades educativas especiales de sus alumnos a su práctica.

Por último el docente realiza su trabajo en una institución determinada con una comunidad educativa concreta. La evaluación docente debería señalar la existencia de una orientación y asesoramiento profesional al alumno en competencias interprofesionales, cómo va a ser la comunicación con las familias y la participación de estas en el proceso de aprendizaje de los alumnos. Y además conocer las relaciones con la gestión y organización del centro escolar y el entorno.

La tercera dimensión incluida es el desarrollo profesional docente, se analiza la formación docente. La participación en formación formal y no formal y su reconocimiento. La existencia de iniciativas de cooperación para establecer redes de aprendizaje. Los resultados de la evaluación docente deberían definir las necesidades de desarrollo de los docentes (Eurydice, 2018).

Los diferentes instrumentos de recogida de información de la evaluación docente son variados, como por ejemplo: la observación en el aula, el cumplimiento de objetivos o entrevistas individuales. En ocasiones la autoevaluación docente incluye realizar un portafolio. Puede aplicarse también un sistema estandarizado con un rango de dimensiones 
establecidas. Otros instrumentos son el resultado académico de los alumnos o encuestas a familias y alumnos.

Quién realiza esta recogida de datos, quiénes son los evaluadores son elementos a tener en cuenta en el análisis. Hay un amplio abanico de agentes: el mismo docente, inspectores externos de la administración, el equipo directivo o los pares (TALIS, 2018).

Toda evaluación tiene sentido si se trasladan los datos obtenidos. Se han tenido en cuenta como indicadores si se trata de una evaluación formativa que capacite al docente hacia las buenas prácticas. Y sumativa en relación a avance en la carrera docente, decisiones en momentos clave, recompensas por rendimiento o sanciones a docentes ineficaces (TALIS, 2018).

Tabla 1.

Árbol de Parámetros de la evaluación docente.

(Siguiente página ->) 


\begin{tabular}{|c|c|c|c|}
\hline \multicolumn{4}{|c|}{ ÁRBOL DE PARÁMETROS: EVALUACIÓN DOCENTE } \\
\hline DIMENSIONES & PARÁMETROS & \multicolumn{2}{|c|}{ INDICADORES } \\
\hline \multirow{12}{*}{$\begin{array}{l}\text { CARÁCTER DE LA } \\
\text { EVALUACIÓN }\end{array}$} & \multirow{2}{*}{ OBLIGATORIEDAD } & \multicolumn{2}{|l|}{ Obligatoria } \\
\hline & & \multicolumn{2}{|c|}{ Voluntaria (promoción dentro de la carrera) } \\
\hline & \multirow{5}{*}{ PROFESORES } & \multicolumn{2}{|c|}{ Todos } \\
\hline & & \multirow{4}{*}{$\begin{array}{l}\text { Subconjunto } \\
\text { de profesores } \\
\text { en función de... }\end{array}$} & Tipo de contrato \\
\hline & & & Etapa de la carrera \\
\hline & & & Nivel de educación \\
\hline & & & $\begin{array}{l}\text { Tipo de educación (programas generales, } \\
\text { programas vocacionales educación adultos...) }\end{array}$ \\
\hline & \multirow{2}{*}{ NATURALEZA } & \multicolumn{2}{|c|}{$\begin{array}{l}\text { Interna (propia escuela elabora sus instrumentos y los } \\
\text { resultados revierten en ella). }\end{array}$} \\
\hline & & \multicolumn{2}{|c|}{ Externa (instrumentos estandarizados) } \\
\hline & \multirow{3}{*}{ FRECUENCIA } & \multicolumn{2}{|c|}{ Intervalos regulares } \\
\hline & & \multicolumn{2}{|c|}{ Etapas clave de la carrera (promoción) } \\
\hline & & \multicolumn{2}{|c|}{ Ocasiones específicas (final periodo de pruebas o contrato) } \\
\hline \multirow{20}{*}{$\begin{array}{l}\text { EVALUACIÓN DE LA } \\
\text { PRÁCTICA DOCENTE }\end{array}$} & \multirow{3}{*}{$\begin{array}{l}\text { PLANIFICACIÓN Y } \\
\text { PREPARACIÓN }\end{array}$} & \multicolumn{2}{|c|}{ Objetivos de la enseñanza } \\
\hline & & \multicolumn{2}{|c|}{$\begin{array}{l}\text { Conocimiento del currículo: contenidos del área o áreas que } \\
\text { imparte }\end{array}$} \\
\hline & & \multicolumn{2}{|c|}{$\begin{array}{l}\text { Competencias pedagógicas en la docencia de la propia } \\
\text { asignatura: didáctica del área que imparte }\end{array}$} \\
\hline & EVALUACIÓN & \multicolumn{2}{|c|}{$\begin{array}{l}\text { Evaluación de estudiantes y práctica de evaluación: sistema de } \\
\text { la evaluación del proceso aprendizaje con retroalimentación al } \\
\text { alumno }\end{array}$} \\
\hline & & \multicolumn{2}{|c|}{ Evaluación de la enseñanza } \\
\hline & & \multicolumn{2}{|c|}{$\begin{array}{l}\text { Crear ambiente de respeto en un marco multicultural y } \\
\text { multilingüe }\end{array}$} \\
\hline & CLIMA DEL AULA & \multicolumn{2}{|c|}{$\begin{array}{l}\text { Establecer una cultura de aprendizaje desde un enfoque } \\
\text { competencial }\end{array}$} \\
\hline & & \multicolumn{2}{|c|}{ Comportamiento de estudiantes y gestión de una clase. } \\
\hline & & \multicolumn{2}{|c|}{ Organización del espacio físico } \\
\hline & & \multicolumn{2}{|c|}{$\begin{array}{l}\text { Métodos de aprendizaje individualizado, involucrando a los } \\
\text { alumnos en su propio aprendizaje. }\end{array}$} \\
\hline & & \multicolumn{2}{|c|}{ Trabajo cooperativo } \\
\hline & METODOLOGÍA & \multicolumn{2}{|c|}{ Competencias transversales de docencia } \\
\hline & & Competencias d & gitales para el aprendizaje y para la enseñanza \\
\hline & & Proyectos de In & ovación \\
\hline & & Investigación er & el aula \\
\hline & $\begin{array}{l}\text { NECESIDADES } \\
\text { EDUCATIVAS } \\
\text { ESPECIALES }\end{array}$ & $\begin{array}{l}\text { Métodos de ens } \\
\text { alumnos con NI }\end{array}$ & $\begin{array}{l}\text { ñanza inclusivos para dar respuesta a los } \\
\text { E }\end{array}$ \\
\hline & & $\begin{array}{l}\text { Orientación y as } \\
\text { competencias in }\end{array}$ & $\begin{array}{l}\text { esoramiento profesional al alumno en } \\
\text { terprofesionales }\end{array}$ \\
\hline & RELACIONES CON & Relaciones con & as familias \\
\hline & $\begin{array}{l}\text { LA COMUNIDAD } \\
\text { EDUCATIVA }\end{array}$ & Gestión y Admi & istración de la escuela \\
\hline & & $\begin{array}{l}\text { Relaciones con } \\
\text { entorno }\end{array}$ & entorno: contribuir con la escuela y el \\
\hline
\end{tabular}

(Continua ->) 


\begin{tabular}{|c|c|c|}
\hline \multirow{3}{*}{$\begin{array}{l}\text { DESARROLLO } \\
\text { PROFESIONAL } \\
\text { DOCENTE }\end{array}$} & $\begin{array}{l}\text { FORMACIÓN } \\
\text { DOCENTE }\end{array}$ & Participación en formación formal y no formal \\
\hline & COOPERACIÓN & Cooperación-Redes de aprendizaje \\
\hline & RECONOCIMIENTO & Reconocimiento de la formación \\
\hline \multirow{11}{*}{$\begin{array}{l}\text { RECOGIDA DE } \\
\text { DATOS DE LA } \\
\text { EVALUACIÓN }\end{array}$} & \multirow{7}{*}{$\begin{array}{l}\text { INSTRUMENTOS DE } \\
\text { EVALUACIÓN DE LA } \\
\text { PRÁCTICA DOCENTE }\end{array}$} & Observación en el aula \\
\hline & & Establecimiento de objetivos y entrevistas individuales \\
\hline & & Autoevaluación del docente \\
\hline & & Portafolio docente \\
\hline & & Forma estandarizada en un rango de dimensiones \\
\hline & & Resultados estudiantiles \\
\hline & & Encuestas padres, alumnos. \\
\hline & \multirow{4}{*}{ EVALUADORES } & Profesores-autoevaluación \\
\hline & & Inspectores externos \\
\hline & & Equipo directivo \\
\hline & & Revisión por pares \\
\hline \multirow{5}{*}{$\begin{array}{l}\text { DEVOLUCIÓN DE } \\
\text { RESULTADOS DE } \\
\text { LA EVALUACIÓN } \\
\text { DOCENTE Y SU USO }\end{array}$} & FORMATIVA & $\begin{array}{l}\text { Capacitación del docente hacia buenas prácticas y eliminación } \\
\text { de malas prácticas. }\end{array}$ \\
\hline & \multirow{4}{*}{ SUMATIVA } & Avance en la carrera \\
\hline & & Decisiones en momentos clave de la carrera \\
\hline & & Recompensas de rendimiento \\
\hline & & Sanciones a docentes ineficaces \\
\hline
\end{tabular}

Fuente: elaboración propia

\section{Desarrollo de la investigación}

Una vez elaborado el árbol de parámetros, se seleccionarán los aspectos referentes a la evaluación de la práctica educativa. Se busca una imagen contextualizada de cada uno de los países a estudiar en la fase descriptiva e interpretativa de la investigación. Posteriormente se expone lo que se ha derivado de una fase de yuxtaposición y comparativa.

En la fase descriptiva el primer país que se analizó fue Alemania. Alemania está dividida en 16 estados federales o Länder, cada estado es el encargado de legislar y administrar el sistema educativo alemán. Sin embargo, la política educativa de todo el país está coordinada por la Kulturministerkonferenz (KMK) formada por todos los Ministros de Educación y Cultura de los diferentes Länder. Se puede decir que existe una Ley fundamental (Grundgesetz) que se concreta luego en los diferentes Länder (OCDE, 2014).

Los centros educativos están administrados por las autoridades locales, en general son públicos, aunque también hay un porcentaje de instituciones religiosas y privadas. Las escuelas poseen una autonomía pedagógica que las autoridades deben respetar. La influencia del estado se ejerce a través de la aprobación de programas específicos. La supervisión de la escuela es ejercida por la inspección educativa (Schulinspecktion) con una evaluación externa que supervisa el desarrollo de la calidad educativa.

El segundo país analizado fue España. En España las competencias educativas están transferidas a las diferentes comunidades autónomas, son estas las encargadas de elaborar los planes de evaluación. La norma general recoge la participación del profesorado (LOE, 2006). Los planes serán públicos y recogerán los fines y criterios de valoración y la 
participación de la comunidad educativa. En España hay tres tipos de instituciones educativas según su financiación o titularidad. Pueden ser públicas, privadas concertadas donde la enseñanza es subvencionada por el estado y privada sin concierto (OCDE, 2014).

En tercer lugar, Finlandia, donde el Ministerio de Educación y Cultura de Finlandia no realiza ningún tipo de evaluación. La evaluación docente se lleva a cabo a partir de objetivos, proyectos y compromisos de la escuela. Es la dirección del centro la responsable de desarrollar políticas que considere efectivas. Esto implica una supervisión sobre el trabajo del docente y su contratación. Las autoridades locales supervisan que los planes de mejora o proyectos se ejecuten y su impacto en la mejora de la escuela. La dirección de la escuela supervisa pedagógicamente el trabajo docente, que se presupone profesional y con las competencias docentes necesarias para la autoevaluación y la mejora (OCDE, 2013).

Por último, se analizó el modelo anglosajón. El Reino Unido está compuesto por cuatro países (Escocia, Gales, Inglaterra e Irlanda del Norte) cada uno de ellos tiene competencias en el área educativa aunque existen similitudes en el sistema educativo. El pasado junio de 2016 los ciudadanos ingleses votaron a favor de la salida de Reino Unido de la UE, se ha mantenido por su singularidad y porque a nivel educativo aún no se conoce como afectará el Brexit. Son los equipos directivos los que ejercen el liderazgo pedagógico, ya que el contexto educativo es de una creciente autonomía escolar. La evaluación docente es diferente en los cuatro países, así en Irlanda del Norte, Escocia y Gales está más centrada en la autoevaluación, mientras que en Inglaterra son evaluaciones externas a partir de estándares (OCDE, 2015). En septiembre de 2012, Inglaterra introdujo un sistema de evaluación racionalizado relacionado con los estándares de competencia y conducta. Este marco tiene la finalidad de concretar cómo los docentes deben desarrollar su desempeño desde su cualificación inicial. Recoge una autoevaluación y la reflexión sobre ella como elemento para la mejora de la práctica docente. Esta evaluación de la propia práctica está contrastada con la retroalimentación de los colegas Qualified Teacher Status (OCDE, 2015).

\section{Discusión, conclusiones y limitaciones}

El análisis comparativo de estas cuatro realidades europeas ofrece un espectro muy amplio de evaluación de la práctica docente. A pesar de las recomendaciones de la Comisión Europea no se puede concluir que las competencias docentes requeridas actualmente estén presentes totalmente, en dichos modelos de evaluación. Tal vez los sistemas que más se acercan son el de Reino Unido y el finlandés. El primero mediante una valoración estandarizada y el segundo con una regulación local, por tener incorporada la cultura evaluativa como elemento de mejora en las prácticas docentes (Education Departament, 2009). El caso en el que la evaluación de la práctica docente es casi insuficiente y con un desarrollo desigual, es España, aunque hay comunidades autónomas que han desarrollado planes de evaluación como Castilla-La Mancha, Navarra y Cantabria que supervisan con regularidad el sistema de evaluación (Eurydice, 2018). Podemos poner como excepción dentro del sistema español, la supervisión realizada sobre los funcionarios en prácticas.

Con respecto al carácter de la evaluación es en el Reino Unido (Inglaterra) donde se encuentra más estructurada y sistematizada, siendo obligatoria para todos los docentes y combinando la orientación interna y externa a intervalos regulares anuales. Esto viene generado por la estructura escolar es de cuasi mercado y es la escuela la encargada de la contratación del docente y de los resultados de la institución (OCDE, 2015). Además 
incluyen un apartado de comportamiento personal y profesional que abarca el ámbito emocional del docente (Qualified Teacher Status, 2013).

En contraposición está el caso finlandés que no posee ningún tipo de estructuración con respecto a la evaluación docente. Lo paradógico es que en Finlandia la cultura de la evaluación como elemento de mejora de la práctica docente está incluida en el desempeño docente, recogen competencias en la definición de escuelas comprensivas (Isoré, 2009). Son las propias escuelas las que realizan esta valoración a partir de unos objetivos a los que se comprometen como instituciones, anualmente es revisado con la administración local. Los docentes son contratados por la escuela (Eurydice, 2018).

Una organización intermedia es el caso de Alemania, donde se da una evaluación obligatoria al inicio de la carrera docente. Una vez se accede al funcionariado, las prácticas de avaluación docente son voluntarias, aunque por los informes internacionales consultados no poseen mucha relevancia (OCDE-Indikatoren: Germany, 2017). La administración educativa realiza evaluaciones externas y hay procesos de autoevaluación en las escuelas que supervisan la calidad de la enseñanza en las instituciones educativas (Eurydice, 2018). En los últimos tiempos tras la reforma de 2008, para unificar la educación en todos sus Länder, ha intentado incluir estos aspectos evaluativos dentro de las dinámicas de los centros. En 2008 se encontraron grandes carencias en la formación continua docente y el desarrollo de pedagogías activas que revertiesen en la mejora de los aprendizajes de los alumnos (Kotthoff, 2013). Se están intentado implementar modelos de evaluaciones que funcionan en algunos Ländern, ya que la cultura evaluativa empieza a tomar relevancia a pesar de la oposición por parte de algunos sectores educativos como los sindicatos de profesores (Odendahl, 2017).

En España es la inspección la que, de forma puntual, supervisa bajo una rúbrica si durante la práctica docente se recogen esas competencias. Suele ser en momentos puntuales, como el acceso a la carrera docente o como seguimiento de un plan de mejora en determinado centro escolar. Es el país en el que más proporción del profesorado trabaja en centros en los que nunca han sido evaluados (TALIS, 2018). No se trata de una práctica regular y no existe un marco general que regule su aplicación (Eurydice, 2018).

Todos los sistemas buscan incorporar a la valoración del desarrollo profesional docente, porque es la formación continua la que facilita al docente las estrategias para enfrentarse a las necesidades formativas de la sociedad actual (Eurydice, 2018). Es en Alemania donde menos repercusión tiene, es cierto que la formación inicial docente en este país es de gran calidad, pero no se mantiene en el tiempo. En Finlandia se recoge como requisito del centro educativo participar en formaciones, proyectos de cooperación con otras escuelas formando redes de aprendizaje, una formación contextualizada con las necesidades de la escuela y sus objetivos como institución (Eurydice, 2018). Los estándares ingleses recogen este apartado de formación docente como iniciativa y versatilidad dentro de las competencias docentes requeridas. Por último, en España esta formación docente posee un reconocimiento de méritos y es imprescindible para obtener el complemento salarial de antigüedad (Eurydice, 2018).

Los instrumentos para la recogida de datos son diferentes en función de la naturaleza de la evaluación. En la evaluación interna, se suele realizar a una autoevaluación donde se recoge la práctica docente en un portafolio que facilita la reflexión, en el caso finlandés (Sahlberg, 2010). Cuando la evaluación es de naturaleza externa se utiliza la observación en el aula por pares (otros docentes, inspectores o dirección) los cuatro sistemas educativos utilizan en algún momento este instrumento. En ocasiones la observación se recoge en un rango de dimensiones de forma estandarizada. 
En los sistemas educativos donde la contratación depende de las administraciones locales o del propio centro, las entrevistas individualizadas suponen otro elemento de recogida de información como en el caso de Finlandia o Reino Unido. En Alemania la evaluación docente tiene repercusiones para el acceso a nuevas responsabilidades.

Los resultados estudiantiles, de una u otra manera forman parte de la evaluación del centro o de los docentes, ya sea de forma explícita o implícita.

El país donde la evaluación docente posee un mayor carácter formativo es Finlandia (Sahlberg, 2010), es en los otros tres sistemas donde adquiere un carácter más sumativo. La combinación de una evaluación interna mediante la práctica reflexiva o autoevaluación combinada con una evaluación externa ya sea estandarizada o por cumplimiento de objetivos como en el caso finlandés se dibuja como la opción más eficaz. Ya que repercute directamente en el proceso de enseñanza-aprendizaje y en la detección de necesidades de los docentes. Este último punto facilita la formación continua enmarcada en un contexto educativo (Education Departament, 2009).

Para conseguir que la evaluación de la práctica docente forme parte del desempeño docente, sería necesario crear una cultura evaluativa como búsqueda de la mejora y del rigor (Marina, Pellicer y Manso, 2015). Cuando no existe esta cultura ayudaría establecer unos estándares, en función de las competencias docentes necesarias para facilitar esta evaluación. Una evaluación con un plan de mejora y con seguimiento.

Con respecto a los instrumentos de recogida de datos es recomendable que combinasen aspectos cuantitativos y cualitativos para tener una visión más acertada de la realidad. Este análisis debe recoger las 28 "Core Competences" y su desarrollo en el aula y el contexto escolar.

Se abren dos campos de trabajo para incorporar las Core Competences. Por un lado en la formación inicial y por otro en la formación continua docente, donde se incorporen las competencias no solo referidas al dominio de la materia y la pedagogía sino también al desarrollo personal del docente. Profundizando en las habilidades sociales necesarias para gestionar el clima del aula, el desarrollo de interacciones sociales desde la equidad y el respeto en una sociedad multicultural, multilingüe. Formación para atender a la diversidad de necesidades desde este enfoque y construir una sociedad con los valores democráticos (Valle, y Manso, 2017). Es el modelo evaluativo de Inglaterra el que atiende a ese aspecto personal del docente para atender a aspectos personales de los alumnos y colabore al buen clima del centro escolar.

La evaluación resulta eficiente a la dinámica de los centros cuando es sistemática y la devolución de resultados repercute en el desarrollo docente. La aportación de las observaciones por pares contrastada con la autoevaluación reflexiva y combinada con los resultados de los alumnos genera una triangulación positiva en la práctica docente.

Un modelo de evaluación dinámico, alejado de ser un mero control burocrático que incida en la mejora continua del docente y la institución en la que participa genera una escuela viva, capaz de aprender de sus propios errores. El objetivo principal es seguir aprendiendo de forma individual y colectiva desde un desarrollo profesional y personal. Crear una cultura evaluativa que dé lugar a procesos de evaluación dinámicos que influyan directamente sobre el aprendizaje de los alumnos, el desarrollo profesional docente, considerando este como un elemento necesario dentro de la práctica docente a todos los niveles (Marina, Pellicer y Manso, 2015).

Una de las limitaciones del estudio es que no queda claro, que los sistemas de evaluación docente de los diferentes países incluyan las destrezas docentes recomendadas por la Comisión Europea para lograr la eficacia educativa. 
Las preguntas que surgen son ¿cuáles son las limitaciones para no desarrollar este tipo de prácticas? ¿Por qué la evaluación del desempeño no se percibe como elemento enriquecedor de la práctica y la mejora? No se tiene la certeza de que la mala praxis no se dé. ¿Cómo se podría detectar?

\section{Referencias}

Bolívar, A. (2008). Evaluación de la práctica docente. Una revisión desde España. RIEE. Revista Iberoamericana de Evaluación Educativa. 1(2). https://revistas.uam.es/ riee/article/view/4666

Calderhead, J., Denicolo, P., \& Day, C. (2012). Research on teacher thinking (RLE Edu N): Understanding professional development. Routledge. https://doi. org/10.4324/9780203126578

Cardús Ros, S. (2007). El desconcierto de la educación las claves para entender el papel de la familia, la escuela los valores, los adolescentes, la televisión...y la inseguridad del futuro. Ediciones B.

Comisión Europea (2012b). A Supporting the Teaching Professions for Better Learning Outcomes Accompanying the document Communication from the Commission "Rethinking Education: Investing in skills for better socio-economic outcomes". Estrasburgo, SWD (2012) 374 final. http://eurlex.europa.eu/LexUriServ/ LexUriServ.do?uri=SWD:2012:0374:FIN:EN:PDF

Comisión Europea/Eacea/Eurydice (2008). Autonomía y responsabilidades del profesorado en Europa Informe Eurydice. Bruselas.http://doi.org/10.2766/78890

Comisión Europea/EACEA/Eurydice (2015). La profesión docente en Europa: prácticas, percepciones y políticas. Oficina de Publicaciones de la Unión Europea. http:// doi.org/10.2797/63946

Comisión Europea/EACEA/Eurydice (2018). La profesión docente en Europa: Acceso, progresión y apoyo. Oficina de Publicaciones de la Unión Europea. https://doi. org $/ 10.2797 / 708723$

Cortés, Á. C., Manso, J., Matarranz, M., \& López, J. M. V. (2016). Investigación en Educación Comparada: Pistas parainvestigadores noveles. Revistalatinoamericana de Educación comparada, 7(9), 39-56.

Darling-Hammond, L. (2000). Teacher quality and student achievement. Education policy analysis archives, 8 , 1. https://doi.org/10.14507/epaa.v8n1.2000

Darling-Hammond, L., Wei, R. C., Andree, A., Richardson, N., \& Orphanos, S. (2009). Professional learning in the learning profession. National Staff Development Council, 12.

Day, C. (2002). Developing Teachers: The challenges of lifelong learning. Routledge.

Education Departament (2009). Appendix A. Assessment of Personal Performance, City of Helsinki. 
Esteve, J. M. (2003). La tercera revolución educativa: La educación en la sociedad del conocimiento. Paidós.

European Comission (2004). Common European principles for teacher competences and qualifications. Brussels: Directorate-General for Education and Culture. Available at: http://www.see-educoop.net/education_in/ pdf/o1-en_principles_ en.pdf.

European Comission. (2010). Developing coherent and system-wide Induction Programmes for beginning Teachers. A Handbook for policymeakers. Luxemburgo: Directorate-General for Education and Culture. http://ec.europa. eu/education/library/publications/handbooko410_en.pdf.

European Comission. (2013). Study on Policy Measures to improve the Attractiveness of the Teaching Profession in Europe. Luxemburgo: Oficina de Publicaciones de la Unión Europea.http://ec.europa.eu/education/library/study/2013/teachingprofession1_en.pdf.

European Commission; Directorate-General for Communication. (2019). Documento de reflexión para una Europa sostenible de aquí a 2030. Luxembourg. https://ec.europa.eu/info/publications/reflection-paper-towards-sustainableeurope-2030_es

EURYDICE (2004). The Teaching Profession in Europe: Profile, Trends and Concerns (Report III: Working Conditions and Pay). Bruselas: Eurydice. Disponible en: http://www.eurydice.org.

EURYDICE (2013). Cifras clave del profesorado y la dirección de centros educativos en Europa. Edición 2013. Informe de Eurydice. Luxemburgo: Oficina de Publicaciones de la Unión Europea. http://eacea.ec.europa.eu/education/eurydice/documents/ key_data_series/151ES.pdf.

Gil, L. (2005). Los sistemas educativos europeos y la formación de profesores: Los casos de Francia, Reino Unido, España y Finlandia. Revista entreideias: educação, cultura e sociedade, 10(9).

Isoré, M. (2009). Teacher Evaluation: Current Practices in OECD Countries and a Literature Review, OECD Education Working Papers, No. 23, OECD Publishing, Paris. http://dx.doi.org/10.1787/223283631428.

Kotthoff, H. G. (2013). 'New 'solutions To'old'problems? Recent Reforms In Teacher Education In Germany/ ¿'Nuevas' soluciones a los' viejos' problemas? Reformas recientes en la formación del profesorado en Alemania. Revista Española de Educación Comparada, (22), 73-92.

Ley Orgánica 2/2006, de 3 de mayo, de Educación. Boletín oficial del Estado, 106(4), 17158-17207.

Marcelo García, C. (2002). Aprender a enseñar en la sociedad del conocimiento. Education Policy Analysis Archives, 1-50. 
Marina, J., Pellicer, C. y Manso, J. (2015). Borrador del Libro blanco de la profesión docente y su entorno escolar. Madrid. http://www.mecd.gob.es/mecd/dms/mecd/ destacados/libro-blanco-profesion-docente.pdf.

Montero Lagos, P. (2004). Roles para la docencia universitaria concordantes con las demandas educacionales del nuevo siglo. Reencuentro (Mexico City, Mexico), (40),

Odendahl, W. (2017). Bildungskrise-PISA and the German Educational Crisis. Journal of education, 209.

OECD (2009). Teacher Evaluation. A Conceptual Framework and examples of Country Practices. Recuperado de: www.oecd.org/edu/school/44568106.pdf

OECD (2010). Specific Policy Recommendations on the Development of a Comprehensive In-Service Teacher Evaluation Framework. Recuperado de http://www.oecd.org/ edu/school/48481142.pdf

OECD (2013), Teachers for the 21st Century: Using Evaluation to Improve Teaching, International Summit on the Teaching Profession, OECD Publishing, Paris, https:// doi.org/10.1787/9789264193864-en.

OECD (2014a). Resultados de TALIS 2013: Una perspectiva internacional sobre la enseñanza y el aprendizaje, TALIS, Publicaciones de la OCDE, París, https://doi. org/10.1787/9789264196261-en

OECD (2014b) Education Policy Outlook Spain. OECD Publishing, París, https://www. oecd.org/education/highlightsspain.htm

OECD (2015). Education Policy Outlook 2015: Making Reforms Happen, OECD Publishing, Paris, https://doi.org/10.1787/9789264225442-en.

OECD (2019), TALIS 2018 Results (Volume I): Teachers and School Leaders as Lifelong Learners, TALIS, OECD Publishing, Paris, https://doi.org/10.1787/1dobc92a-en.

OECD (2020). Education Policy Outlook in Germany, OECD Education Policy Perspectives, No. 16, OECD Publishing, Paris, https://doi.org/10.1787/47b795b1-en.

Palacios González, J. (1990). Enseñar y aprender en el contexto del aula. Desarrollo Psicológico y Educación, 2, 357-386.

Sahlberg, P. (2010). The secret to Finland's success: Educating teachers. Stanford Center for Opportunity Policy in Education, 2, 1-8. https://webgate.ec.europa.eu/fpfis/ mwikis/eurydice/index.php/Teachers_and_Education_Staff

Sánchez-Tarazaga, L. (2016). Los marcos de competencias docentes: Contribución a su estudio desde la política educativa europea. Journal of supranational policies of education.

Sebarroja, J. C. (2007). La educación y la escuela ante los cambios sociales. In $E l$ desarrollo de competencias docentes en la formación del profesorado (pp. 9-32). Secretaría General Técnica.

Valle, J. \& Manso, J. (2017). Lifelong Teacher Education; Ser docente a lo largo de la vida. Sial Pigmalión. 